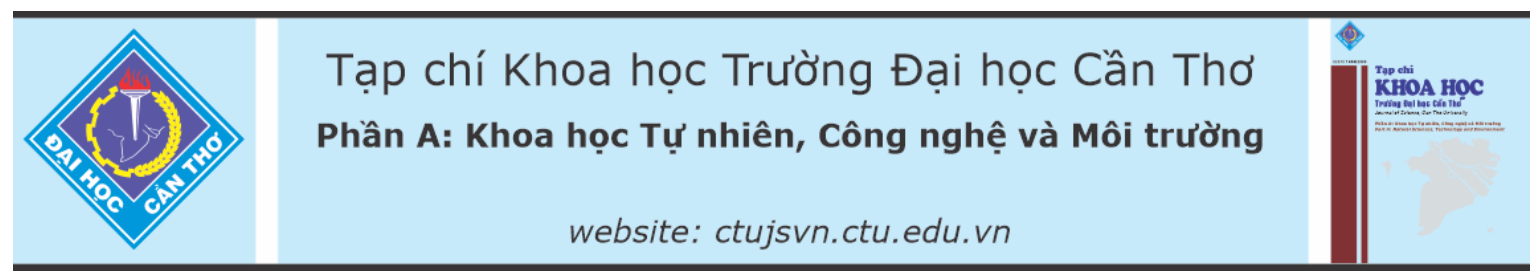

DOI:10.22144/ctu.jvn.2021.172

\title{
XÁC ĐỊNH DIỆN TÍCH LÁ VÀ TÁN LÁ BÀNG PHẦM MỀM IMAGEJ VỚI HİNH CHỤP CỦA IPHONE 6S PLUS
}

\author{
Mai Ngọc Dũng ${ }^{1 *}$ và Tạ Ánh Minh ${ }^{2}$ \\ ${ }^{1}$ Khoa Su phạm Khoa hoc Tư nhiên, Truờng Đại họ Sài Gòn \\ ${ }^{2}$ Trung tâm Giống Cây trồng, Vật nuôi và Thủy sản, Thành phố Hồ Chí Minh \\ *Ngưòi chịu trách nhiệm về bài viết: Mai Ngọc Dũng (email: maingocdung@sgu.edu.vn)
}

\section{Thông tin chung:}

Ngày nhận bài: 04/07/2021

Ngày nhận bài sủa: 17/08/2021

Ngày duyệt đăng: 25/12/2021

Title:

Measurement of leaf area and canopy by imagej software and digital image of iPhone 6s Plus

\section{Tù khóa:}

Chrysanthemum sp., dạng lá, hình kỹ thuật số, ô chuẩn

\section{Keywords:}

Calibration area, Chrysanthemum sp., digital image, leaf shape

\begin{abstract}
The paper was to determine the calibration area of square, leaf area and canopy area by photograph of iPhone 6s Plus and measurement of ImageJ software, without a need for the leaf scanners and functional program. Seven calibration areas of square with different sizes $\left(4-196 \mathrm{~cm}^{2}\right)$ and color (purple, green and red) and different heights of camera phone $(25-125 \mathrm{~cm})$, digital image of fourteen leaves with various shapes and sizes, the canopy different ages of Chrysanthemum sp. were selected for study. The results, the overall average relative error between area measurement by ImageJ and calibration area of square were $0.9 \%, y=1.0005 x+0.2262$ and $R^{2}=0.9987$. The fourteen leaf surface area of various shapes which were $4.6 \%$ of $\% \mathrm{CV}, y$ $=0.9902 x+2.1802$ and $R^{2}=0.9953$. In addition, it has applied to determine the canopy of Chrysanthemum, increased canopy level from the one week old to eight weeks old which was from $152 \pm 18$ to $4026 \pm 978 \mathrm{~mm}^{2}$. It was concluded that iPhone 6s Plus and ImageJ software were sufficient for leaf area and canopy measurements. This result is particularly useful for studying crop physiology.
\end{abstract}

\section{TÓM TẮT}

Nghiên cưu xác định diện tích các ô chuẩn, diện tích bề măt lá và tán lá bẳng hình chup của iPhone 6s Plus và đo bằng phần mềm ImageJ mà không cần sử dụng thiết bi quét lá chuyên dụng cùng với phần mềm tương ưng. Vật liệu nghiên cứu gồm bảy ô vuông chuẩn với kích thuơớc tù 4 đển $196 \mathrm{~cm}^{2}$ với màu tím, xanh lá cây và đỏ cùng với chiều cao chup tù 25 đến $125 \mathrm{~cm}$, hình chup của 14 loại lá với hình dạng và kích thuớc khác biệt, tán lá cây cúc 'Chrysanthemum sp. 'có độ tuổi khác nhau. Kết quả nghiên cứu đã xác định mức độ sai số thực tế giữa diện tích các ô chuẩn hình vuông do ImageJ tính với diện tích các ô chuẩn là 0,9\%, $y=1,0005 x+$ 0,2262 và $R^{2}=0,9987$. Diện tích bề mạt lá của 14 dạng lá khác nhau với $\%$ CV là 4.6\%, $y=0,9902 x+2,1802$ và $R^{2}=0,9953$. Ngoài ra, đề tài đã úng dụng xác định diện tích tán lá cây cúc tù 1 tuần tuổi tới 8 tuần với kết quả diện tích tán lá tăng tù $152 \pm 18$ đến $4026 \pm 978 \mathrm{~mm}^{2}$. iPhone 6s Plus và phần mềm ImageJ có thể thục hiện xác định diện tích lá và tán lá. Kết quả nghiên cứu có thể hũu dụng trong nghiên cứu về sinh lí cây trồng. 


\section{GIỚI THIẸU}

Diện tích bề mặt lá là một trong những chỉ tiêu xác định sự phát triển của thực vật trong quá trình tăng trưởng và là thành tố quan trọng trong công thức tính chỉ số diện tích lá (LAI 'leaf area index'). Xác định bề mặt lá có rất nhiều ứng dụng trong các ngành Sinh lí thực vật, Nông học, Lâm học, Sinh thái ... Các công trình nghiên cứu xác định bề mặt lá bằng phương pháp trực tiếp, gián-trực tiếp và gián tiếp cùng với việc ứng dụng kỹ thuật số để phân tích vẫn đang tiếp tục nghiên cứu. Các thiết bị quét chuyên dụng thường được sử dụng như CI202 Portable Laser Leaf Area Meter, LICOR LI3000 leaf area meter, Leaf Area Meter, OKI MB451 scanner... (Agehara et al., 2020; Dogan et al., 2018) và dùng để quét hình lá cùng với phần mềm kèm theo có giá thành cao. Nghiên cứu mô phỏng chỉ số diện tích lá (LAI) lúa từ mô hình Oryza 2000 tại các điểm canh tác lúa của tỉnh Sóc Trăng của nhóm tác giả Khoa Môi trường \& Tài nguyên Thiên nhiên thuộc Trường Đại học Cần Thơ đã sử dụng máy quét hình (không nêu số hiệu) để xác định diện tích bề mặt lá (Phạm Thị Lệ Huyền \& Võ Quang Minh, 2014). Lê Văn Trọng (2016) đã sử dụng máy đo diện tích lá CI - 202 của Hoa Kỳ để xác định diện tích bề mặt lá khi nghiên cứu một số chỉ tiêu sinh lí, hóa sinh của 10 giống đậu phộng (Arachis hypogaea L.) trồng tại Thanh Hóa. Nghiên cứu về chỉ số diện tích bề mặt lá $(\mathrm{LAI})$ cho các giai đoạn phát triển khác nhau của cà chua và dưa leo với các phương pháp xử lí nước tưới khác nhau, các tác giả sử dụng AccuPAR ceptometer LP-80 và Leaf Area Software để xác định LAI (Hossain et al., 2017). Diện tích bề mặt lá của 70 mẫu cây đã được xác định bằng việc sử dụng phần mềm OpenCV trên smartphone chạy bởi hệ điều hành Android, kết quả thí nghiệm được so sánh với thiết bị chuyên dụng Lab's leaf area meter dựa trên khổ giấy A4 làm chuẩn (Hariadi et al., 2018).

Drienovsky et al. (2017) đã xây dựng phần mềm xác định diện tích bề mặt lá 'Scan LeafArea', nhóm đã thực nghiệm trên 3 loài lá cây khác nhau thuộc cây thân gỗ và so sánh với phần mềm ImageJ, kết quả khác biệt không đáng kể giữa phần mềm của tác giả với ImageJ. Khi nghiên cứu sự phát triển tán lá của Arabidopsis thaliana để phân tích diện tích tán lá, Srikanth et al. (2017) đã sử dụng phần mềm ImageJ V1.49 để tính diện tích tán lá nhưng không có ghi nhãn hiệu camera. Nghiên cứu sự phát triển tán lá rừng ngập mặn vùng Wallacea, vịnh Bone thuộc miền Nam Sulawesi tại Indonesia, Mursalim et al. (2020) đã sử dụng ImageJ để phân tích sự phát triển tán lá của rừng ngập mặn từ 1999 - 2018 từ không ảnh của vệ tinh Landsat 7 cung cấp. Kết quả xác định diện tích tán lá trên cánh đồng dựa vào hình kỹ thuật số của smartphone và sử dụng ImageJ phân tích tổng diện tích tán lá của tác giả thuộc Trường Đại học Florida (Agehara, 2020) cùng với việc xác định diện tích bề mặt lá trực tiếp trên cây. Ngoài ra, nhóm tác giả đã so sánh kết quả đo diện tích 100 lá hoa bia và 100 lá hồ tiêu giữa thiết bị quét ảnh chuyên dụng LI-3100C với phần mềm ImageJ, kết quả phân tích không có khác biệt đáng kể (Agehara et al., 2020). Ngoài ra, phần mềm ImageJ được nhóm tác giả Wang et al. (2018) ứng dụng trong việc đo chiều cao và độ che phủ của hệ thống cây trồng trong đô thị vùng Harbin thuộc tỉnh Heilongjiang Trung Quốc. Nhóm tác giả sử dụng thước chuẩn chiều cao là $1 \mathrm{~m}$ và chiều ngang lòng đường theo đơn vị mét làm chuẩn. Kết quả không có sự khác biệt khi xác định chiều cao của cây.

Phần mềm ImageJ của viện Sức khỏe Hoa Kỳ cung cấp miễn phí, cập nhật liên tục và là chương trình ứng dụng được sử dụng khá phổ biến trên thế giới để nghiên cứu quá trình quang hợp, sự phát triển tán lá, độ che phủ của cây trồng... Ngoài ra, ImageJ có thể ứng dụng trong nghiên cứu vi sinh vật như đếm số lượng tế bào bán tự động, thiết lập thanh tỷ lệ trên hình ảnh tự động ... (National Institutes of Health, 2021).

Mục đích của nghiên cứu là xác định chiều cao hữu dụng và phù hợp khi chụp hình các ô chuẩn để tạo thuận tiện chụp hình lá và tán cây ngoài thực địa bằng iPhone $6 \mathrm{~s}$ Plus cùng với việc xác định diện tích bằng phần mềm ImageJ. iPhone $6 \mathrm{~s}$ Plus là thiết bị di động nhỏ gọn sẽ giúp việc thu thập dữ liệu hình ảnh dễ dàng với chất lượng tốt $(1080$ x 1920 pixel) tại thực địa và có thể thay thế các thiết bị quét hình chuyên dụng đắt tiền. Hình ảnh có thể phân tích trên các phần mềm khác nhau và đặc biệt là ImageJ. Tuy nhiên, kết quả xác định diện tích phải qua hai bước là chụp hình và phân tích trên ImageJ trong khi các thiết bị chuyên dụng sẽ xác định diện tích một cách trực tiếp.

\section{VÂT LIỆU VÀ PHƯƠNG PHÁP NGHIẾN CÚU}

\subsection{Phương tiện, thiết bị và vật liệu nghiên cứu}

Thiết bị được sử dụng là iPhone $6 \mathrm{~s}$ Plus và ứng dụng chức năng camera - grid với mục đích là mẫu vật được chụp sẽ nằm vào khoảng trung tâm của khung hình và bề mặt của điện thoại sẽ song song với mặt phẳng mẫu vật. iPhone $6 \mathrm{~s}$ Plus có 1 camera phía sau với độ phân giải là $8 \mathrm{MP}$, khẩu độ là $\mathrm{f} / 2,2$, 
kích thước điểm ảnh là $1,5 \mu$ với sự hỗ trợ công nghệ đặc biệt là chống rung quang học OIS 'optical image stabilization' và chỉ có phiên bản iPhone $6 \mathrm{~s}$ Plus trở lên.

Đề tài sử dụng phần mềm ImageJ V1.52p để xác định diện tích các ô chuẩn (màu và diện tích khác nhau), các dạng lá khác nhau và tán lá cây cúc theo từng thời điểm phát triển.

Vật liệu nghiên cứu là các ô chuẩn màu tím, màu xanh lá và màu đỏ với các diện tích khác nhau là 4 , $16,36,64,100,144$ và $196 \mathrm{~cm}^{2}$. Mười bốn dạng lá khác nhau về hình thái được thu nhận tại khu vực Q8 thành phố Hồ Chí Minh. Sự phát triển tán lá cây cúc 'Chrysanthemum $s p$.' từ 1 tuần tuổi đến 8 tuần tuổi được khảo sát tại Phòng Kiểm nghiệm, thử nghiệm giống cây trồng, vật nuôi và thủy sản thuộc Trung tâm Giống cây trồng, vật nuôi và thủy sản, Sở Nông nghiệp và Phát triển nông thôn Thành phố Hồ Chí Minh tại 4A181 đường Thanh Niên ấp 4, xã Phạm Văn Hai, huyện Bình Chánh, thành phố Hồ Chí Minh. Thời gian khảo sát từ ngày 20/8/2019 (ngày chuyển cây cúc cấy mô sang khay và chuyển ra vườn ươm) đến 11/12/2019.

\subsection{Phương pháp nghiên cứu}

2.2.1. Xác định sai số thực tế của các ô chuẩn với giá trị phân tích hình ảnh của ImageJ

Các diện tích ô chuẩn gồm $4,16,36,64,100$, 144 và $196 \mathrm{~cm}^{2}$ với màu tím $(\lambda=400-450 \mathrm{~nm})$, màu xanh lá cây $(\lambda=495-570 \mathrm{~nm})$ và màu đỏ $(\lambda=$ $620-750 \mathrm{~nm}$ ) cùng với thước chuẩn sẽ được chụp tại các độ cao là $25,50,75,100$ và $125 \mathrm{~cm}$ (Muller-Linow et al., 2019). Hình sẽ được phân tích trên ImageJ: Threhold - Default and Red. (Baecker, 2015; Bankhead, 2014; Easlon \& Bloom, 2014). Xác định đường chuẩn giữa các diện tích ô chuẩn với chiều cao khác nhau (Aboukarima et al., 2017; Dogan et al., 2018; Espinosa et al., 2010; Hariadi et al., 2018) với mục dích là xác định chiều cao hữu dụng và phù hợp để chụp hình. Diện tích các ô chuẩn gần như tương ứng với diện tích các dạng lá trong nghiên cứu, màu các ô chuẩn sẽ đại diện cho phổ ánh sáng khả kiến $(\lambda=400-700 \mathrm{~nm})$ thuộc vùng màu $\mathrm{RGB}$ của hai biên và vùng giữa của phổ ánh sáng khả kiến.

\subsubsection{Xác định diện tích bề mặt các dạng lá và tán lá cây cúc 'Chrysanthemum sp."}

Mười bốn dạng lá khác nhau và sự phát triển tán lá cây cúc 'Chrysanthemum sp. 'từ 1 tuần đến 8 tuần tuổi sẽ được chụp và phân tích trên ImageJ (Agehara, 2020; Das et al., 2016; Dogan et al., 2018). Hình 14 dạng lá được đặt trên nền giấy trắng và sử dụng lệnh Image, Type, 8-bit để chuyển màu dạng RGB của hình sang dạng hình 8-bit với thang màu xám và sử dụng lệnh Threhold - Default and Red để xác định phạm vi vùng phân ngưỡng màu đỏ của lá cây trên ảnh phân tích của ImageJ. Hình các tán lá được chụp tại thực địa và sẽ phân tích bằng lệnh Image, Type, Lab Stack để chuyển màu dạng RGB của hình sang dạng hình có thang màu xám, các màu tím, xanh lá cây cùng với màu đỏ sẽ chuyển sang thang màu đen. Lệnh Threhold - Default and Red sẽ chuyển thang màu đen thành màu đỏ để xác định phạm vi vùng phân ngưỡng màu đỏ của ImageJ (Broeke et al., 2015; Mohamed et al., 2019). Diện tích mẫu vật được thực hiện bởi lệnh Analyze, Tools, ROI Manager và sử dụng Wand tool xác định vị trí mẫu vật cần đo diện tích.

\subsubsection{Phân tích và thống kê số liệu}

Các mẫu sẽ được đo 3 lần lấy giá trị trung bình \pm độ lệch chuẩn, xác định hệ số biến thiên $\% \mathrm{CV}$ 'Coefficient of variation' với ngưỡng giới hạn là nhỏ và bằng $20 \%$. Hệ số biến thiên là một thước đo thống kê độ phân tán của các dữ liệu trong một chuỗi dữ liệu so với giá trị trung bình. Phân loại \%CV được dựa vào độ phân tán và gồm 4 mức độ: Thấp là $<10 \%$; Trung bình là $\geq 10 \%$ và $<20 \%$; Cao $\geq$ $20 \%$ và $<30 \%$; Rất cao là $\geq 30 \%$ (Couto et al., 2013).

Công thức tính hệ số biến thiên

$$
\% \mathrm{CV}=\frac{\sigma}{\mu} 100
$$

Với: $\sigma$ là độ lệch chuẩn và $\mu$ là giá trị trung bình.

Phân tích số liệu bằng Multiple Range Tests với độ tin cậy $95 \%$ với $\mathrm{p}<0,05$ bằng phần mềm Statgraphics Centurion XVII.II - X64 - USA.

\section{KẾT QUẢ VÀ THẢO LUẬN}

Hình chụp mẫu vật được sử dụng chức năng camera - grid của iPhone 6s Plus với mục đích tạo mặt phẳng song song với mẫu vật. Sử dụng phầm mềm ImageJ V1.52p để xác định diện tích các ô chuẩn, các dạng lá khác nhau và tán lá của lá cây cúc 'Chrysanthemum sp.' từ 1 tuần tuổi tới 8 tuần của hình chụp. Các kết quả tính diện tích được phân tích trên phần mềm thống kê Statgraphics Centurion XVII.

\subsection{Xác định sai số thực tế của các ô chuẩn với giá trị phân tích hình ảnh của ImageJ}

Các ô chuẩn hình vuông có màu tím $(\lambda=400-$ $450 \mathrm{~nm})$, màu xanh lá cây $(\lambda=495-570 \mathrm{~nm})$ và 
màu đỏ $(\lambda=620-750 \mathrm{~nm})$, các ô màu có diện tích chuẩn là $4,16,36,64,100,144$ và $196 \mathrm{~cm}^{2}$ được chụp hình tại các độ cao từ camera của iPhone $6 \mathrm{~s}$ Plus tới mẫu vật là $25,50,75,100$ và $125 \mathrm{~cm}$. Kết quả xác định diện tích trung bình và sai số thực tế các ô chuẩn khi phân tích của ImageJ được trình bày tại Bảng 1.

Bảng 1. Diện tích trung bình và sai số thực tế các ô chuẩn của ImageJ

\begin{tabular}{|c|c|c|c|c|c|c|c|}
\hline \multirow[b]{2}{*}{ Độ cao } & \multicolumn{7}{|c|}{ Diện tích các ô chuẩn $\left(\mathrm{cm}^{2}\right)$} \\
\hline & \multicolumn{7}{|c|}{ Màu xanh lá } \\
\hline$(\mathbf{c m})$ & $\mathbf{4 , 0}$ & 16,0 & $\mathbf{3 6 , 0}$ & 64,0 & 100,0 & 144,0 & 196,0 \\
\hline 25 & $4,1 \pm 0,06$ & $16,8 \pm 0,17$ & $35,4 \pm 0,29$ & $65,8 \pm 1,00$ & $103,0 \pm 1,76$ & $141,0 \pm 5,77$ & $190,7 \pm 1,92$ \\
\hline 50 & $4,0 \pm 0,06$ & $16,0 \pm 0,32$ & $35,7 \pm 0,72$ & $63,4 \pm 1,25$ & $99,9 \pm 1,97$ & $139,9 \pm 2,76$ & $195,0 \pm 2,75$ \\
\hline 75 & $4,0 \pm 0,10$ & $16,3 \pm 0,36$ & $37,3 \pm 0,81$ & $63,7 \pm 1,42$ & $100,8 \pm 2,18$ & $146,8 \pm 3,21$ & $197,3 \pm 1,69$ \\
\hline 100 & $4,1 \pm 0,10$ & $16,5 \pm 0,12$ & $37,0 \pm 0,23$ & $64,4 \pm 0,40$ & $100,8 \pm 0,58$ & $147,1 \pm 0,87$ & $196,6 \pm 1,54$ \\
\hline \multirow[t]{2}{*}{125} & $4,1 \pm 0,07$ & $16,3 \pm 0,25$ & $36,8 \pm 0,61$ & $64,2 \pm 1,03$ & $100,7 \pm 1,64$ & $146,5 \pm 2,35$ & $195,1 \pm 0,81$ \\
\hline & \multicolumn{7}{|c|}{ Màu đỏ } \\
\hline 25 & $4,2 \pm 0,12$ & $16,8 \pm 0,12$ & $35,0 \pm 0,29$ & $65,4 \pm 0,52$ & $104,9 \pm 0,87$ & $144,3 \pm 1,50$ & $189,2 \pm 2,02$ \\
\hline 50 & $\pm 0,21$ & $16,2 \pm 0,17$ & $35,6 \pm 0,50$ & & $\pm 1,04$ & $5 \pm 1,44$ & $8 \pm 2,02$ \\
\hline 75 & $4,2 \pm 0,06$ & $\pm 0,36$ & $36,5 \pm 0,81$ & $\pm 1,31$ & $3 \pm 2,17$ & $3 \pm 3,18$ & $5 \pm 4,29$ \\
\hline 100 & $4,2 \pm 0,06$ & $15,9 \pm 0,31$ & $36,1 \pm 0,70$ & $62,9 \pm 1,25$ & $99,2 \pm 1,90$ & $5 \pm 2,86$ & $0 \pm 2,47$ \\
\hline \multirow[t]{3}{*}{125} & $4,2 \pm 0,10$ & $16,0 \pm 0,40$ & $36,2 \pm 0,91$ & $63,2 \pm 1,57$ & $99,5 \pm 2,42$ & $9 \pm 2,70$ & $199,1 \pm 2,62$ \\
\hline & \multicolumn{7}{|c|}{ Màu tím } \\
\hline & 4,0 & 16,0 & 36,0 & 64,0 & 100,0 & 144,0 & 196,0 \\
\hline 25 & $4,3 \pm 0,06$ & $16,6 \pm 0,35$ & $37,1 \pm 0,40$ & $64,9 \pm 1,33$ & $7 \pm 1,91$ & $2 \pm 0,58$ & $4 \pm 0,46$ \\
\hline 50 & 0,15 & 0,20 & $\pm 0,55$ & 64 & $\pm 1,45$ & $\pm 2,00$ & $\pm 2,75$ \\
\hline 75 & 0,06 & $\pm 0,21$ & $37,6 \pm 0,49$ & $64,0 \pm$ & $100,8 \pm 1,34$ & $\pm 1,49$ & $4 \pm 1,91$ \\
\hline 100 & 0,26 & $\pm 0,91$ & $36,2 \pm 2,16$ & $61,7 \pm 3,71$ & $\pm 5,73$ & $\pm 8,62$ & $9 \pm 6,21$ \\
\hline \multirow[t]{3}{*}{125} & $2 \pm 0,06$ & $16,2 \pm 0,15$ & $36,9 \pm 0,36$ & $63,1 \pm 0,57$ & $98,2 \pm 0,91$ & $145,6 \pm 1,32$ & $199,6 \pm 1,70$ \\
\hline & \multicolumn{7}{|c|}{ Giá trị trung bình các ô chuẩn $\left(\mathrm{cm}^{2}\right)$} \\
\hline & $4,1 \pm 0,13$ & $16,2 \pm 0,43$ & $36,4 \pm 0,98$ & $63,8 \pm 1,56$ & $100,6 \pm 2,71$ & $144,7 \pm 3,63$ & $196 \pm 4,07$ \\
\hline & \multicolumn{7}{|c|}{ Sai số thực tế (\%) } \\
\hline & $2,50 \%$ & $1,30 \%$ & $1,10 \%$ & $-0,30 \%$ & $0,60 \%$ & $0,50 \%$ & $0,00 \%$ \\
\hline \multicolumn{8}{|c|}{ Hệ số \% CV } \\
\hline & $3,20 \%$ & $2,70 \%$ & $2,70 \%$ & $2,40 \%$ & $2,70 \%$ & $2,50 \%$ & $2,10 \%$ \\
\hline
\end{tabular}

Ghi chú: Độ cao (cm) là chiều cao tù̀ camera của iPhone 6s Plus tới mẫu vật được chụp, \%CV là hệ số biến thiên.

Kết quả phân tích màu của ImageJ đối với các hình chụp các ô chuẩn màu tím, màu đỏ và màu xanh lá (độ cao từ 25 đến $125 \mathrm{~cm}$ ) với các diện tích ô

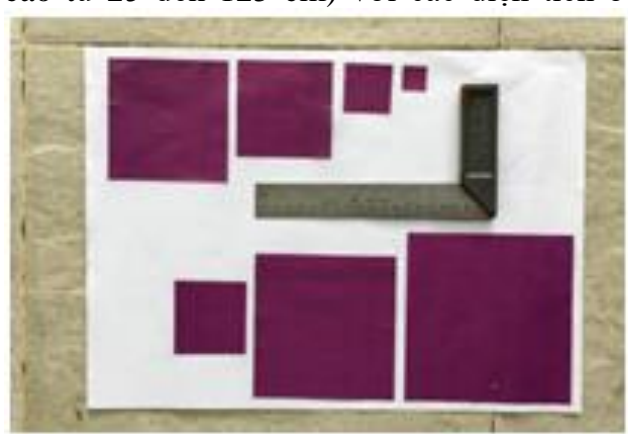

chuẩn là $4,16,36,64,100,144$ và $196 \mathrm{~cm}^{2}$ được trình bày tại Hình 1 , Hình 2 và Hình 3 .

Hình 1. Kết quả phân tích của ImageJ tại độ cao $50 \mathrm{~cm}$ của các ô chuẩn màu đỏ

Ghi chú: Hình bên trái là hình các ô chuẩn màu tím và hình bên phải là ảnh kết quả phân nguõng với màu đỏ thể hiện vùng ảnh được ImageJ xác định là các ô chuẩn chỉ đúng đối với khu vực nền màu trắng (giấy A3). 

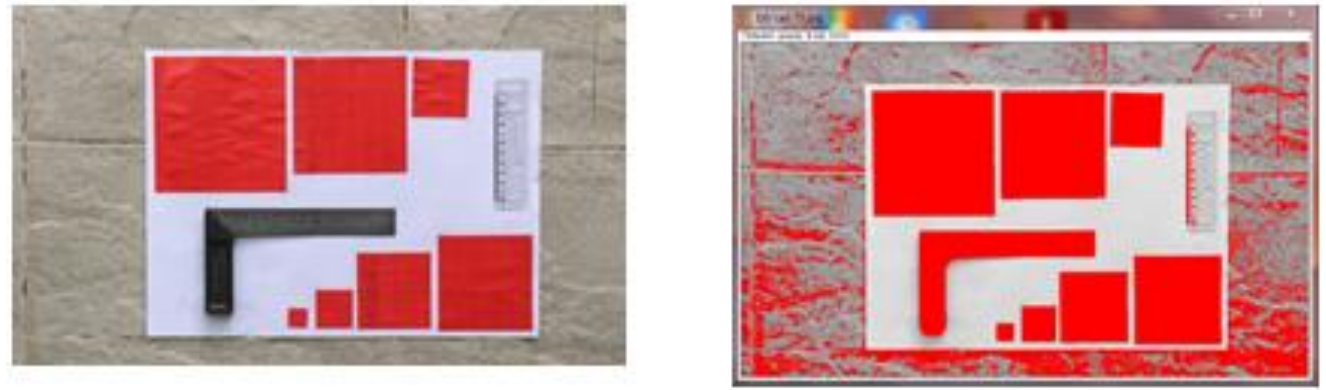

\section{Hình 2. Kết quả phân tích của ImageJ tại độ cao $75 \mathrm{~cm}$ của các ô chuẩn màu đỏ}

Ghi chú: Hình bên trái là hình các ô chuẩn màu đỏ và hình bên phải là ảnh kết quả phân nguõng với màu đỏ thể hiện vùng ảnh được ImageJ xác định là các ô chuẩn chỉ đúng đối với khu vục nền màu trắng (giấy A3).
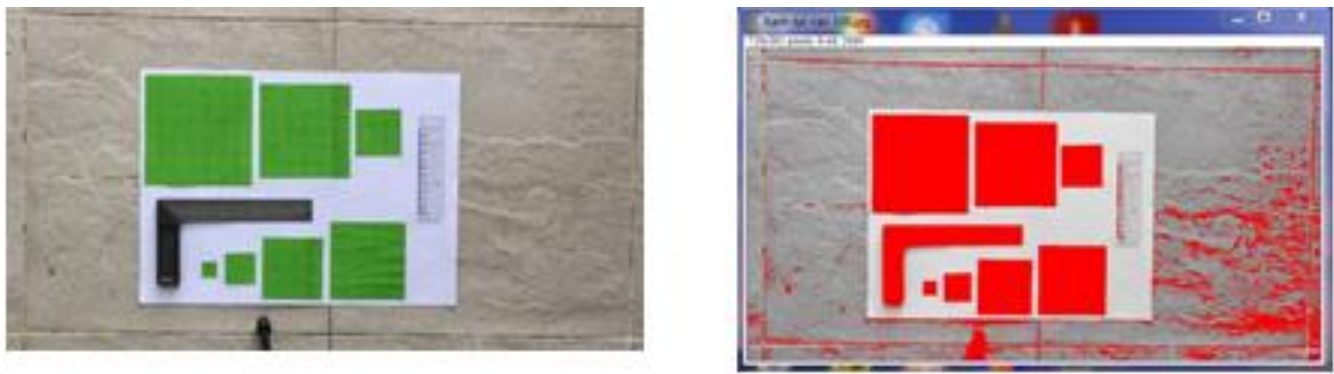

\section{Hình 3. Kết quả phân tích của ImageJ tại độ cao $100 \mathrm{~cm}$ của các ô chuẩn màu xanh lá}

Ghi chú: Hình bên trái là hình các ô chuẩn màu lá cây và hình bên phải là ảnh kết quả phân nguỡng với màu đỏ thể hiện vùng ảnh được ImageJ xác định là các ô chuẩn chỉ đúng đối với khu vực nền màu trắng (giấy A3).

Kết quả xác lập đường chuẩn của các giá trị diện tích trung bình của ô chuẩn ở các màu tím, xanh lá cây và đỏ được đo trên ImageJ tại các độ cao từ 25 $-125 \mathrm{~cm}$ là $\mathrm{y}=1,0005 \mathrm{x}+0,2262$ với $\mathrm{R}^{2}$ là 0,9987 và phụ thuộc vào diện tích ô chuẩn thực tế với các màu tương ứng. Kết quả đã cho thấy ý nghĩa của chức năng camera - grid của iPhone $6 \mathrm{~s}$ Plus và thị trường 'FoV (Field of View)' của camera sẽ tuyến tính theo chiều cao chụp hình. Sai số trung bình thực tế là $0,9 \%$ với hệ số biến thiên dao động từ $2,10-$ $3,2 \%$ và cho phép trong thực nghiệm không cần xác định chiều cao để chụp mẫu vật dao động từ $25 \mathrm{~cm}$ tới $125 \mathrm{~cm}$. Kết quả phân tích giá trị diện tích các ô chuẩn bao gồm Area $4 \mathrm{~S}$, Area $16 \mathrm{~S}, \ldots$, Area $144 \mathrm{~S}$ và Area $196 \mathrm{~S}$ với giá trị diện tích trung bình được tính trên các ô chuẩn tương ứng của ImageJ (Phần mềm Statgraphics Centurion XVII không cho phép nhập liệu bằng Việt ngữ. Vì vậy, các ký hiệu như diện tích ô chuẩn được viết thành Area_S và diện tích đo trên ImageJ được viết thành Area_. Ví dụ: Area $16 \mathrm{~S}$ là diện tích ô chuẩn là $16 \mathrm{~cm}^{2}$ hoặc Area 16 là diện tích ô chuẩn $16 \mathrm{~cm}^{2}$ được đo trên ImageJ). Multiple Range Tests của Statgraphics Centurion XVII đã xác định không có sự khác biệt có ý nghĩa thống kê và mức độ so sánh các cặp 'Homogeneous Groups' là tương đồng. Giá trị nhóm 'Homogeneous Groups' giới hạn từ 0,704849 đến - 0,704849 , các giá trị so sánh giữa Area_S với Area_nằm trong giới hạn cho phép của giá trị khác biệt 'Difference' tuần tự như sau: Area $4 \mathrm{~S}$ - Area 4 là 0,142222 , Area $16 \mathrm{~S}$ - Area 16 là 0,246667 , Area $36 \mathrm{~S}$ - Area 36 là 0,391111 , Area $64 \mathrm{~S}$ - Area 64 là $-0,22$, Area $100 \mathrm{~S}$ - Area 100 là 0,624444 , Area $144 \mathrm{~S}$ - Area 144 là 0,682222 và Area $196 \mathrm{~S}$ - Area 196 là -0,00888889. Kết quả phân tích diện tích ô chuẩn có các màu tím, xanh lá cây và đỏ với độ cao chụp khác nhau cũng cho kết quả không có sự khác biệt có ý nghĩa thống kê và các giá trị nằm trong khoảng cho phép của 'Difference' từ 1,73791 đến $-1,73791$. Đồ thị đường chuẩn các giá trị diện tích trung bình của ô chuẩn của các màu tím, xanh lá cây và đỏ được đo trên ImageJ được trình bày tại Hình 4 . 


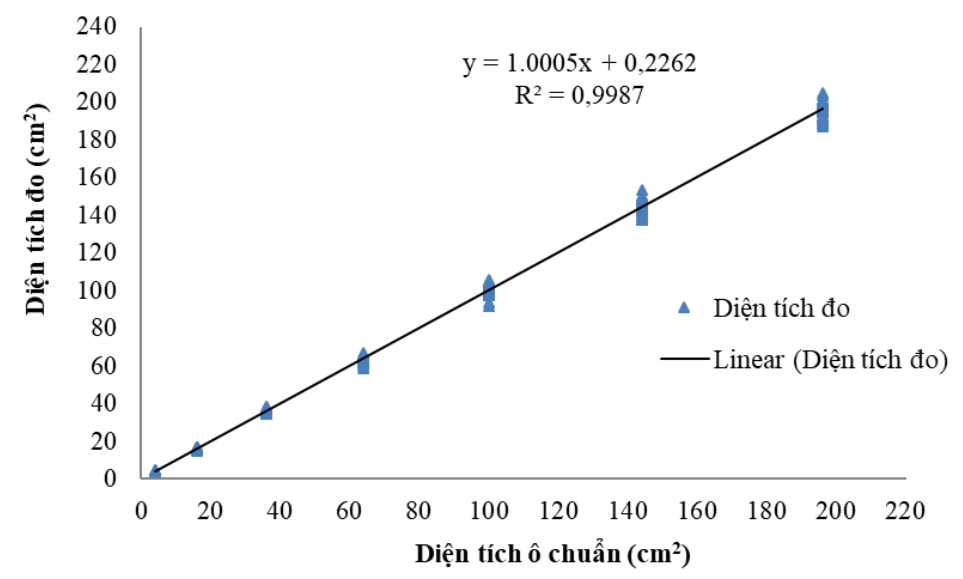

Hình 4. Đồ thị đường chuẩn các giá trị diện tích trung bình của các ô chuẩn

\subsection{Xác định diện tích bề mặt các dạng lá}

Đề tài đã xác định diện tích bề mặt của 14 dạng lá khác nhau và gồm lá kim, các dạng lá đơn, các dạng lá xẻ thùy và các dạng lá kép. Kết quả được trình bày tại Bảng 2 .

Bảng 2. Diện tích trung bình các dạng lá

\begin{tabular}{crrrrr}
\hline Kiểu lá & $\begin{array}{r}\text { Diện tích } \\
\text { lá }\left(\mathbf{c m}^{\mathbf{2}}\right)\end{array}$ & $\begin{array}{r}\text { Hệ số } \\
\mathbf{\%} \mathbf{C V}\end{array}$ & Kiểu lá & $\begin{array}{r}\text { Diện tích } \\
\text { lá }\left(\mathbf{c m}^{\mathbf{2}}\right)\end{array}$ & $\begin{array}{r}\text { Hệ số } \\
\mathbf{\%} \mathbf{C V}\end{array}$ \\
\hline 1A & $150,3 \pm 1,8$ & 1,20 & 8 & $90,1 \pm 4,9$ & 5,44 \\
1B & $88,8 \pm 1,1$ & 1,24 & 9 & $45,9 \pm 2,5$ & 5,45 \\
2 & $51,8 \pm 2,8$ & 5,41 & 10 & $45,1 \pm 2,5$ & 5,54 \\
3 & $53,6 \pm 2,9$ & 5,41 & 11 & $17,0 \pm 1,0$ & 5,88 \\
4 & $30,1 \pm 1,7$ & 5,65 & 12 & $5,7 \pm 0,3$ & 5,26 \\
5 & $223,7 \pm 12,3$ & 5,50 & 13 & $448,3 \pm 14,2$ & 3,17 \\
6 & $40,6 \pm 2,2$ & 5,42 & 14 & $287,1 \pm 7,7$ & 2,68 \\
7 & $198,5 \pm 10,9$ & 5,49 & & & \\
\hline
\end{tabular}

Ghi chú: \%CV là hệ số biến thiên.

Các kiểu lá được thu nhận tại khu vực quận 8 thuộc thành phố Hồ Chí Minh. Hình kỹ thuật số

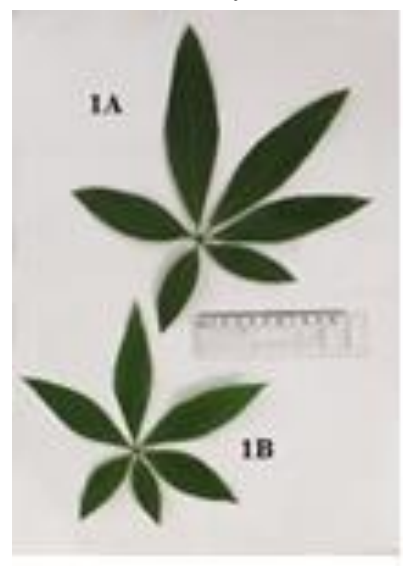

được phân tích trên ImageJ. Kết quả được trình bày tại các Hình $5,6,7$ và 8 .

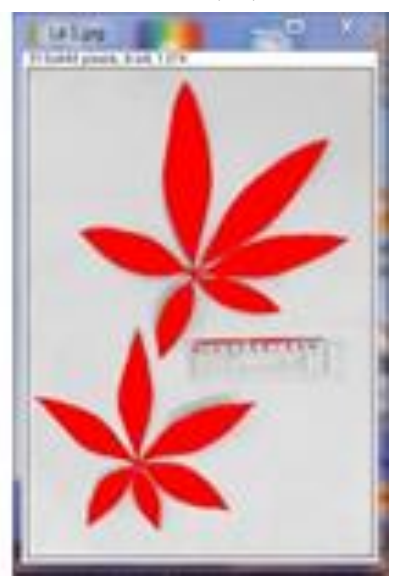

Hình 5. Kiểu lá $1 \mathrm{~A}$ và $1 \mathrm{~B}$ dạng lá phức

Ghi chú: Hình bên trái là hình gốc và hình bên phải là ảnh kết quả phân nguỡng với màu đỏ thể hiện vùng ảnh được ImageJ xác định là lá. 


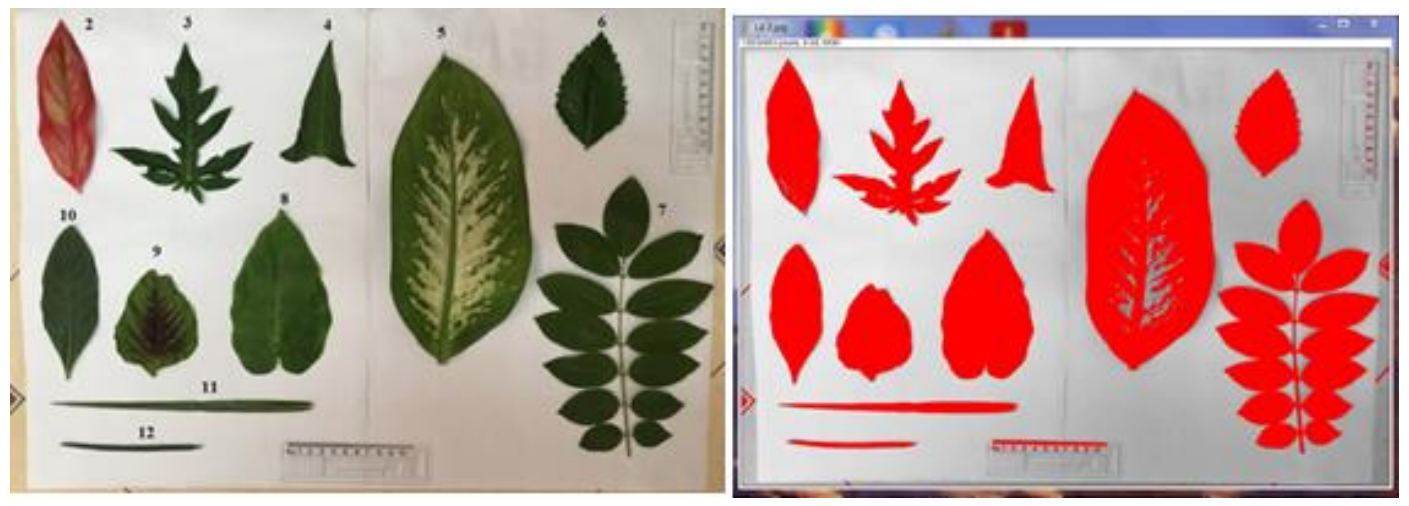

\section{Hình 6. Các kiểu lá khác nhau}

Ghi chú: Kiểu lá đơn gồm 2, 4, 5, 6, 8, 9 và 10. Kiểu lá 3 dạng xẻ thùy. Kiểu lá 7 dạng lá kép. Kiểu lá 11 và 12 dạng lá kim. Hình bên trái là hình gốc và hình bên phải là ảnh kết quả phân nguỡng với màu đỏ thể hiện vùng ảnh được ImageJ xác định là lá.
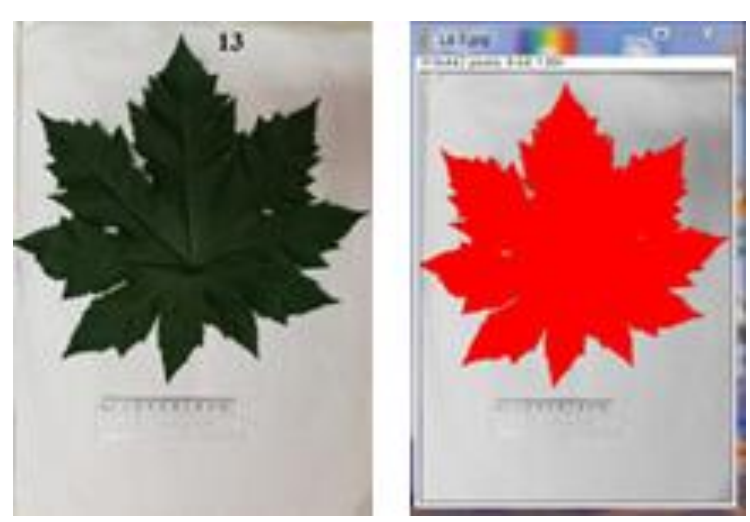

Hình 7. Kiểu lá 13 dạng xẻ thùy
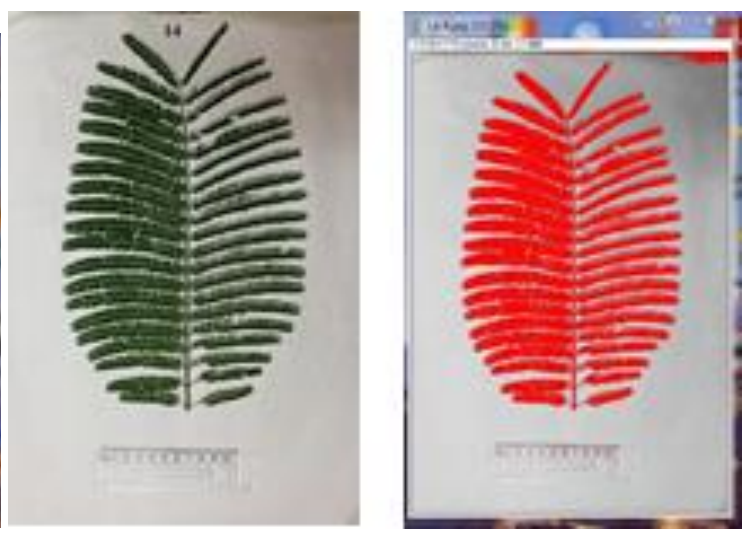

Hình 8. Kiểu lá 14 dạng lá kép lông chim

Ghi chú: Hình bên trái là hình gốc và hình bên phải là ảnh kết quả phân ngương với màu đỏ thể hiện vùng ảnh được ImageJ xác định là lá.

Kết quả xác định mối tương quan giữa các ô chuẩn (màu tím, màu xanh lá và màu đỏ, diện tích từ $\left.4-196 \mathrm{~cm}^{2}\right)$ với diện tích bề mặt của 14 dạng lá khác nhau được phân tích trên ImageJ. Diện tích bề mặt lá của Bảng 2 được sắp xếp lại theo giá trị từ nhỏ tới lớn và gộp các giá trị để tương đương với diện tích ô chuẩn. Diện tích trung bình gộp của các kiểu lá bao gồm kiểu lá $4,6,10,9,2,3$ và kiểu lá $1 \mathrm{~B}, 8$. Kết quả được trình bày tại Bảng 3 và Hình 9 .

Bảng 3. Diện tích trung bình các kiểu lá được gộp chung và diện tích các ô chuẩn tương ứng

\begin{tabular}{llllllllllllll}
\hline Kiểu lá & $\mathbf{1 2}$ & $\mathbf{1 1}$ & $\mathbf{4}$ & $\mathbf{6}$ & $\mathbf{1 0}$ & $\mathbf{9}$ & $\mathbf{2}$ & $\mathbf{3}$ & $\mathbf{1 B}$ & $\mathbf{8}$ & $\mathbf{1 A}$ & $\mathbf{7}$ & $\mathbf{5}$ \\
\hline $\begin{array}{l}\text { Diện tích lá } \\
\left(\mathrm{cm}^{2}\right)\end{array}$ & 5,7 & 17,0 & 30,1 & 40,6 & 45,1 & 45,9 & 51,8 & 53,6 & 88,8 & 90,1 & 150,3 & 198,5 & 223,7 \\
\hline $\begin{array}{l}\text { Diện tích trung bình kiểu lá } \\
\left(\mathrm{cm}^{2}\right)\end{array}$ & 5,7 & 17,0 & & & & 44,5 & 89,5 & 150,3 & 198,5 & 223,7 \\
\hline $\begin{array}{l}\text { Diện tích ô chuẩn } \\
\left(\mathrm{cm}^{2}\right)\end{array}$ & 4,0 & 16,0 & & & & 36,0 & 100,0 & 144,0 & 196,0 & \\
\hline
\end{tabular}




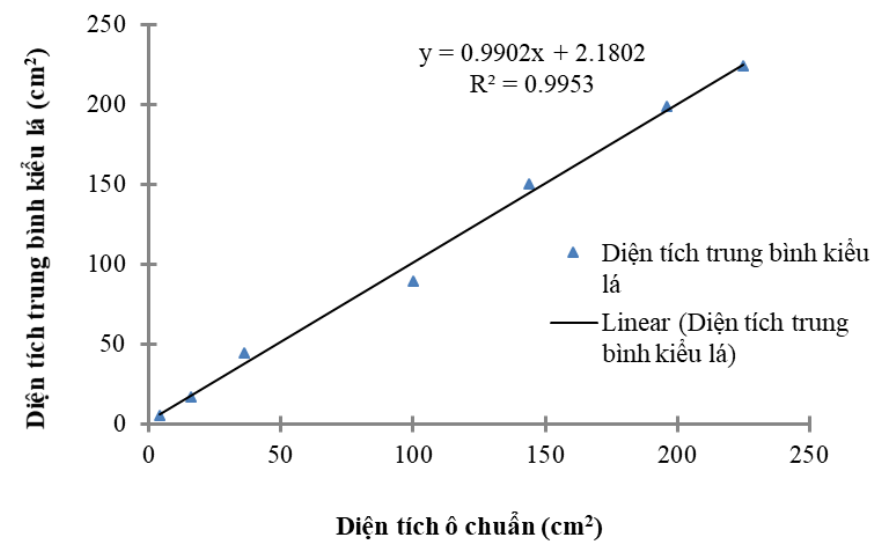

Hình 9. Mối tương quan giữa diện tích lá với diện tích ô chuẩn

Kết quả đã xác lập đường chuẩn $\mathrm{y}=0,9902 \mathrm{x}+$ 2,1802 với $R^{2}$ là 0,9953 . Getman-Pickering et al. (2019) đã sử dụng 16 ô chuẩn được sơn màu đen nhưng không nêu diện tích của ô chuẩn và xác định diện tích 67 lá của 14 loài thực vật khác nhau. Nhóm tác giả đã xác lập được đồ thị đường chuẩn giữa diện tích các ô chuẩn với diện tích bề lá với $\mathrm{R}^{2}$ là 0,9963 khi phân tích trên ImageJ. Agehara et al. (2020) đã thiết lập đường chuẩn của diện tích 100 lá hoa bia và 100 lá hồ tiêu giữa phần mềm ImageJ với thiết bị quét chuyên dụng LI-3100C. Nhóm tác giả đã xác định giá trị tương quan giữa ImageJ với LI-3100C gần bằng $1: 1$ với hàm số $\mathrm{y}=1,02 \mathrm{x}+0,11 ; \mathrm{R}^{2}=$ 0,992 và $\mathrm{y}=1,03 \mathrm{x}+0,06 ; \mathrm{R}^{2}=0,983$.

\subsection{Xác định tán lá cây cúc 'Chrysanthemum $s p$.' từ 1 tuần tuổi đến 8 tuần tuổi}

Đề tài được khảo sát từ ngày 20/8/2019 đến ngày $11 / 12 / 2019$. Ngày 20/8/2019 là giai đoạn cây cúc được cấy mô chuyển sang vườn ươm. Kết quả xác định diện tích tán lá cây cúc từ 1 tuần đến 8 tuần tuồi với $\% \mathrm{CV}$ trung bình là $15,6 \%$ và được trình bày tại Bảng 4 và Hình 10.

Bảng 4. Diện tích tán lá cây cúc 'Chrysanthemum sp.' từ 1 tuần tuổi đến 8 tuần tuổi

\begin{tabular}{|c|c|c|c|c|c|}
\hline $\begin{array}{c}\text { Tuổi cây } \\
\text { (tuần) }\end{array}$ & $\begin{array}{l}\text { Số lượng } \\
\text { cây (cây) }\end{array}$ & $\begin{array}{r}\text { Diện tích } \\
\text { tán lá }\left(\mathrm{mm}^{2}\right) \\
\end{array}$ & $\begin{array}{r}\text { Tuổi cây } \\
\text { (tuần) }\end{array}$ & $\begin{array}{l}\text { Số lượng } \\
\text { cây (cây) }\end{array}$ & $\begin{array}{r}\text { Diện tích } \\
\text { tán lá }\left(\mathrm{mm}^{2}\right)\end{array}$ \\
\hline 1 & 112 & $152 \pm 18$ & 5 & 96 & $1935 \pm 363$ \\
\hline 2 & 110 & $204 \pm 23$ & 6 & 96 & $3526 \pm 633$ \\
\hline 3 & 109 & $685 \pm 61$ & 7 & 96 & $2576 \pm 710$ \\
\hline 4 & 112 & $1235 \pm 43$ & 8 & 96 & $4026 \pm 978$ \\
\hline
\end{tabular}

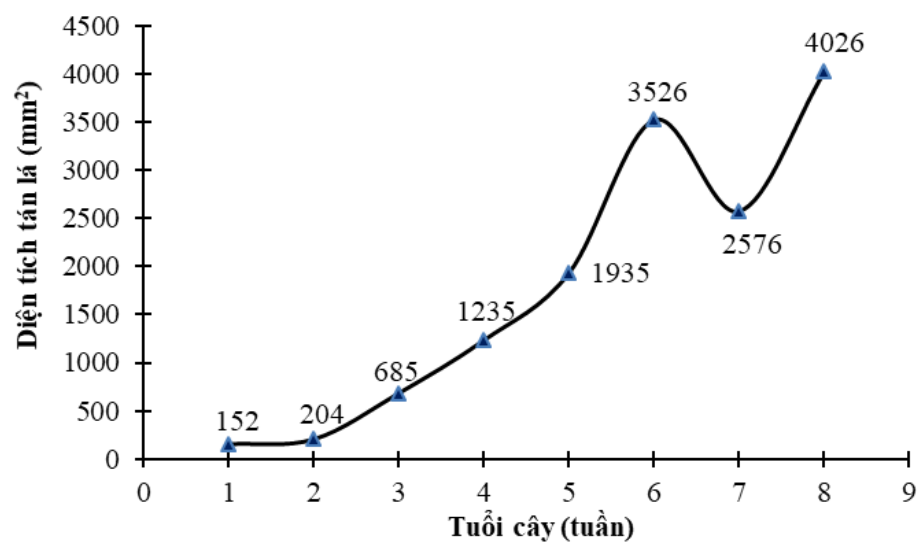

Hình 10. Sự tăng trưởng tán lá cây cúc 'Chrysanthemum sp.' từ 1 tuần tuổi đến 8 tuần tuổi

Ghi chú: Tán lá cây cúc 7 tuần tuổi được tỉa ngọn và nhánh theo quy cách trồng của Trung tâm Giống Cây trồng, Vật nuôi và Thủy sản. 
Kết quả các hình tán lá được chụp bằng iPhone 6s Plus và phân tích trên ImageJ được trình bày tại các Hình $11,12,13,14,15$ và 16 .

Srikanth et al. (2017) khi nghiên cứu sự phát triển tán lá của Arabidopsis thaliana bằng hình kỹ thuật số nhưng không có ghi nhãn hiệu camera và sử dụng phần mềm ImageJ V1.49 để tính diện tích tán lá. Kết quả phân tích đã xác định \% $\%$ trung bình là $18,6 \%$. Mohamed et al (2020) đã nghiên cứu chỉ số diện tích bề mặt lá (LAI) trên cây cà chua và sử dụng ImageJ để tính diện bề mặt lá nhưng không đề cặp tới nhãn hiệu camera, nhóm tác giả sử dụng thước chuẩn hoặc ô chuẩn để quy chuẩn về diện tích bề mặt lá. Kết quả của nhóm tác giả đã xác định mức sai số khá nhỏ là 2,9\%.

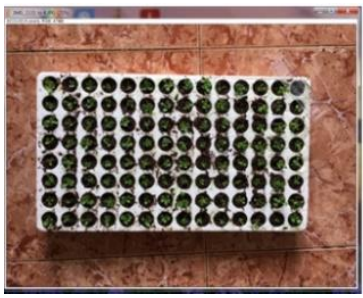

Hình 11. Cây cúc 'Chrysanthemum sp.'

\section{1 tuần tuổi}
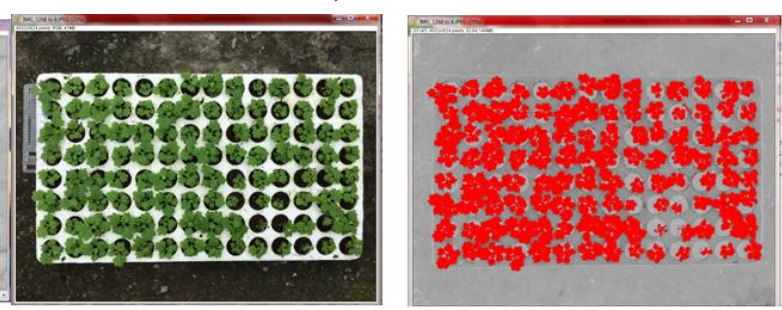

Hình 12. Cây cúc 'Chrysanthemum sp.'

\section{3 tuần tuổi}

Ghi chú: Hình bên trái là hình gốc và hình bên phải là ảnh kết quả phân nguơng với màu đỏ thể hiện vùng ảnh được ImageJ xác định là tán lá.

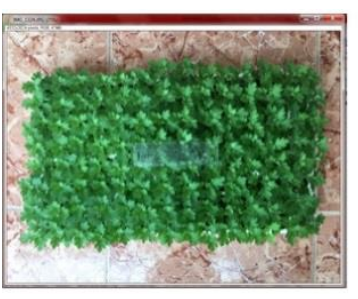

Hình 13. Cây cúc 'Chrysanthemum sp.'

\section{5 tuần tuổi}
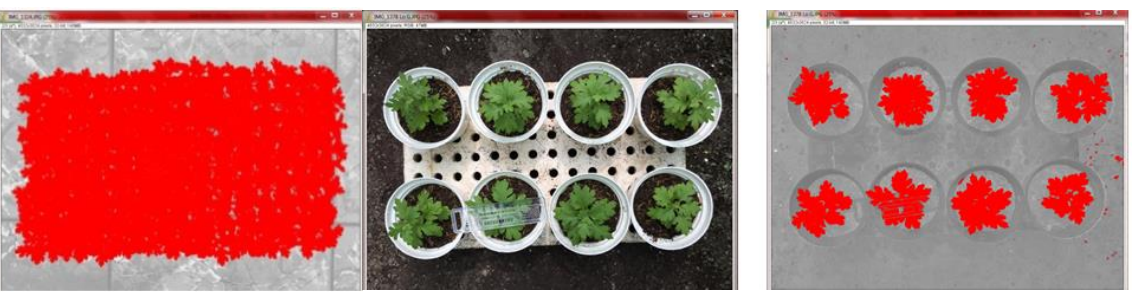

Hình 14. Cây cúc 'Chrysanthemum sp.'

\section{6 tuần tuổi}

Ghi chú: Hình bên trái là hình gốc và hình bên phải là ảnh kết quả phân nguỡng với màu đỏ thể hiện vùng ảnh được ImageJ xác định là tán lá.
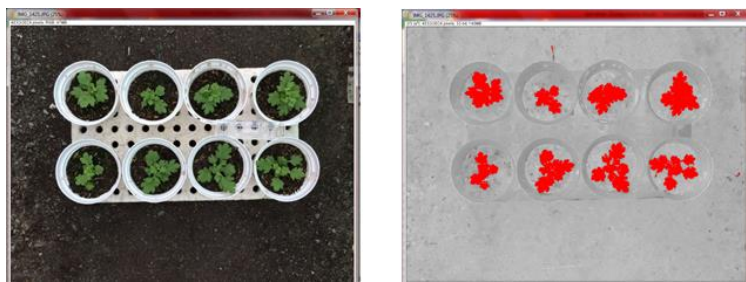

Hình 15. Cây cúc 'Chrysanthemum sp.'

\section{7 tuần tuổi}
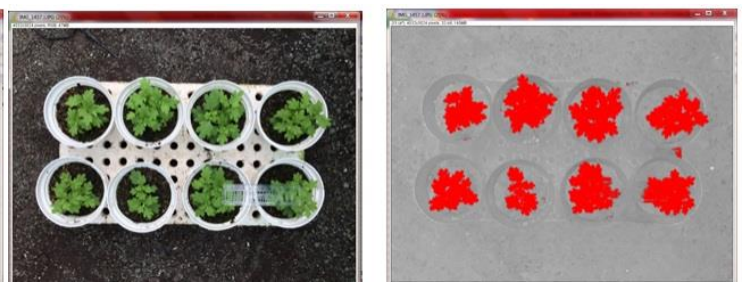

Hình 16. Cây cúc 'Chrysanthemum sp.'

\section{8 tuần tuổi}

Ghi chú: Hình bên trái là hình gốc và hình bên phải là ảnh kết quả phân ngương với màu đỏ thể hiện vùng ảnh được ImageJ xác định là tán lá.

\section{KẾT LUẬN}

Kết quả thí nhiệm đã xác định iPhone 6s Plus với chức năng camera - grid, cùng với tính năng chống rung quang học OIS và phần mềm ImageJ V1.52p có độ tin cậy đạt trên $95 \%$, hệ số biến thiên dao động từ $2,1-3,2 \%$ khi chụp mẫu vật có độ cao từ $25-$
$125 \mathrm{~cm}$ với các ô chuẩn có màu tím $(\lambda=400-450$ $\mathrm{nm})$, màu xanh lá $(\lambda=495-570 \mathrm{~nm})$ và màu đỏ $(\lambda$ $=620-750 \mathrm{~nm}$ ). Kết quả xác định mối tương quan với $R^{2}$ là 0,9953 giữa các ô chuẩn với diện tích bề mặt của 14 dạng lá khác nhau được phân tích trên ImageJ. Sự khác biệt giữa thực nghiệm khi phân tích diện tích lá ImageJ của hình chụp iPhone 6s Plus 
trong nghiên cứu so với các công trình của các tác giả khác gần như tương đương nhau với giá trị $\mathrm{R}^{2}$ gần bằng nhau $\left(\mathrm{R}^{2}=0,99\right)$. $\% \mathrm{CV}$ trung bình đối với hình 14 dạng lá là $4,6 \%$ và hình tán lá cúc từ 1 tuần đến 8 tuần tuổi là $15,6 \%$ khi phân tích trên ImageJ. Giá iPhone $6 \mathrm{~s}$ Plus trên thị trường ở mức trung bình và tiết kiệm ít nhất 7 lần so với máy quét chuyên dụng. Phần mềm ImageJ miễn phí, được cập nhật liên tục và phiên bản cập nhật vào ngày 13/5/2021 là V1.53j. Kết quả nghiên cứu có thể thay thế chức năng quét hình của các thiết bị chuyên dụng đắt tiền và có thể ứng dụng trong việc giảng dạy môn Sinh lí thực vật, nghiên cứu sự phát triển của thực vật, kiểm soát và đánh giá mức độ thiệt hại do dịch bệnh dựa trên việc xác định diện tích, quá trình phát triển của cây trồng trong lãnh vực canh nông khi kết hợp với thiết bị Drone và không ảnh được phân tích trên ImageJ mà các thiết bị quét hình chuyên dụng không có khả năng thực hiện. Các smartphone khác có tính năng và thông số kỹ thuật tương tự iPhone $6 \mathrm{~s}$ Plus cùng với các phiên bản iPhone 7 trở lên có thể sử dụng trong nghiên cứu dựa trên chức năng camera grid và OIS với chiều cao chụp mẫu vật dao động từ $25-125 \mathrm{~cm}$. Tuy nhiên, các camera hoặc smartphone khác cần phải xác lập đường chuẩn giữa giá trị diện tích các ô chuẩn với diện tích các ô chuẩn do ImageJ đo ở các độ cao khác nhau và phải thực hiện hai bước là chụp hình sau đó chuyển hình vào ImageJ phân tích.

\section{TÀI LIỆU THAM KHẢO}

Aboukarima, A. M., Zayed, M. F., Minyawi, M., Elsoury, H. A., \& Tarabye, H. H. H. (2017). Image Analysis-based System for Estimating Cotton Leaf Area. Asian Research Journal of Agriculture, 5(1), 1-8.

Agehara, S. (2020, January 05). Simple imaging techniques for plant growth assessment. https://edis.ifas.ufl.edu

Agehara, S., Pride, L., Gallardo, M., \& HernandezMonterroza, J. (2020, November 08). A simple, inexpensive, and portable image-based technique for nondestructive leaf area measurements. https://edis.ifas.ufl.edu.

Baecker, V. (Ed.). (2015). Workshop: Image Processing and Analysis with ImageJ. Montpellier Ressources Imagerie.

Bankhead, P. (Ed.). (2014). Analyzing fluorescence microscopy images with imagej. Queen's University Belfast.

Broeke, J., Pérez, J. M. M, \& Pascau, J. (2015). Image procession with imagej ( $2^{\text {nd }}$ ed.). Packt Publishing.

Couto, M. F.; Peternelli, L. A. \& Barbosa M. H. P. (2013). Classification of the coefficients of variation for sugarcane crops. Ciência Rural, 43(6), 957-961.

Das, B., Sahoo, R. N., Pargal, S., Krishna, G., Gupta, V. K. R., \& Viswanathan, C. (2016). Measuring leaf area index from colour digital image of wheat crop. Journal of Agrometeorology, 18(1), 22-28.

Dogan, A., Uyak, C., Keskin, N., Akcay, A., Sensoy, R. I. G., \& Ercisli, S. (2018). Grapevine Leaf Area Measurements by Using Pixel Values. Comptes Rendus de l'Academie Bulgare des Sciences, 71(6), 772-779.

Drienovsky, R., Nicolin, A.L., Rujescu, C.\& Sala, F. (2017). Scan leafArea - A software application used in the determination of the foliar surface of plants. Research Journal of Agricultural Science, 49(4), 215-224.

Easlon, H. M., \& Bloom, A. J. (2014). Easy Leaf Area: Automated Digital Image Analysis for Rapid and Accurate Measurement of Leaf Area. Applications in Plant Sciences, 7(2), 1-4.

Espinosa, M. L., Acuña, E. C., Espinosa, M. B., \& Barrera, J. B. (2010). Commercial digital camera to estimate postharvest leaf area index in Vitis vinifera $\mathrm{L}$. Cv. Cabernet sauvignon on a vertical trellis. Chilean Journal of Agricultural Research, 70(2), 315-322.

Getman-Pickering, Z. L., Campbell, A., Aflitto, N., Grele, A., Davis, J. K., \& Ugine, T. A. (2019). LeafByte: A mobile application that measures leaf area and herbivory quickly and accurately. Methods in Ecology and Evolution, 11, 215-221.

Hariadi, T. K., Fadholi, Z., Chamim, A. N., Utama, N. A., Prabasari, I., \& Riyadi, S. (2018). Development of leaf area meter using opencv for smartphone application. Telkomnika, 16(4), 1857-1863.

Hossain, S. A. A. M, Wang, L., Chen, T, \& Li, Z. (2017). Leaf area index assessment for tomato and cucumber growing period under different water treatments. Plant Soil Environment, 10(63), 461-467.

Lê Văn Trọng (2016). Nghiên cứu một số chỉ tiêu sinh lí, hóa sinh của 10 giống lạc (arachis hypogaea L.) trồng tại Thanh Hóa. Tạp chi Khoa học Truòng Đại họ Su phạm Tp Hồ Chí Minh, 6(84), 158-168.

Mohamed, M. M. A., Suliman, A., Puchkov, M., \& Loktionova, E. (2019). Applying a digital method for measuring leaf area index of tomato plants. Atlantis Press, 167, 5-8. https://doi.org/10.2991/ispc-19.2019.2

Muller-Linow, M., Wilhelm, J., Briese, C., Wojciechowski, T., Schurr, U, \& Fiorani, F. (2019). Plant Screen Mobile: An Open-Source Mobile Device App for Plant Trait Analysis. Plant Methods, 15(2), 1-11. 
Mursalim, A., Nurdin, N., Supriad, Nafie, Y. L., Selamat, B., Tresnati, J., \& Tuwo, A. (2020). Mangrove area and vegetation condition resulting from the planting of mangroves in the Wallacea Region, Bone Bay, South Sulawesi. IOP Conf. Series: Earth and Environmental Science, 473.

https://iopscience.iop.org/article/10.1088/17551315/473/1/012055.

National Institutes of Health. USA. (July 6, 2021). https://imagej.nih.gov/ij/docs/intro.html.

Phạm Thị Lệ Huyền \& Võ Quang Minh (2014). Đánh giá khả năng mô phỏng chỉ số diện tích lá lúa bằng phần mềm oryza2000. Tạp chí Khoa học Truờng Đại học Cần Tho, (3), 57-62.

Srikanth, S., Choong, T. W, Chu, J., He, J., \& Chen, Z. (2017). Adaptation of Arabidopsis plants to tropical aeroponics using cool root zone temperatures. American Journal of Plant Sciences, 8(13), 3295-3312.

Wang, W., Xiao, L., Zhang, J., Yang, Y., Tian, P., Wang, H., \& He, X. (2018). Potential of Internet street-view images for measuring tree sizes in roadside forests. Urban Forestry and Urban Greening, 35, 211-220. 